\title{
HYALURONIC ACID 0.2\% APPLICATION ENHANCED PERIODONTITIS TREATMENT IN NON-SURGICAL PHASE
}

\author{
Thuy T. Nguyen' ${ }^{1}$, Hoa T. Ho ${ }^{1}$, Nam C. Huynh', ${ }^{2}$ Vu H.A. Dien ${ }^{3}$, Thuy L. Vo ${ }^{4}$ \\ 'Department of Periodontology, Faculty of Odonto-Stomatology, University of Medicine and Pharmacy at Ho Chi Minh City, Vietnam \\ ${ }^{2}$ Department of Dental Basic Sciences, Faculty of Odonto-Stomatology, University of Medicine and Pharmacy at Ho Chi Minh City, Vietnam \\ ${ }^{3}$ Department of Dental Public Health, Faculty of Odonto-Stomatology, University of Medicine and Pharmacy at Ho Chi Minh City, Vietnam \\ ${ }^{4}$ Department of Implantology, Faculty of Odonto-Stomatology, University of Medicine and Pharmacy at Ho Chi Minh City, Vietnam
}

\begin{abstract}
INTRODUCTION: Topical hyaluronic acid (HA) has been considered as an adjuvant agent for periodontitis (PD). ОвјестіVEs: This study aimed to assess the clinical effects of subgingival application of $0.2 \% \mathrm{HA}$ gel, following scaling and root planning (SRP) in PD patients.

MATERIAL AND METHODS: In this randomized split-mouth study, 733 periodontal pockets of 28 patients with PD were investigated. They were divided into control and experimental groups. The experimental group received subgingival administration of $1 \mathrm{ml} 0.2 \% \mathrm{HA}$ gel into each pocket immediately after SRP, and then after 1 week, 2 weeks, and 3 weeks. Clinical parameters and subgingival plaque were assessed at baseline and 6 weeks after SRP. Quantitative real-time polymerase chain reaction for Porphyromonas gingivalis (Pg), Treponema denticola (Td), Fusobacterium nucleatum ( $F n$ ), and Tannerella forsythia ( $T f$ ) were also performed.

RESULTS: After 6 weeks, there were significant decreases in clinical parameters, and the number of Fn in the experimental group compared to the control group $(p<0.05)$. The number of $P g, T d$, and $T f$ decreased significantly in both groups, with no significant difference between the 2 cohorts $(p>0.05)$.

ConcLusions: The topical application of HA gel in periodontal pockets after SRP may have a beneficial effect in PD patients. This additional treatment appears to help reducing 2 types of bacteria (Tf and Fn) better than non-surgical treatment only.

Trial Registration: Identifier NCT04675385 in ClinicalTrials.gov.
\end{abstract}

KEY WORDS: chronic periodontitis, adjunctive therapy, hyaluronic acid, bactericidal efficiency, clinical parameters.

J Stoma 2021; 74, 2: 76-83

DOI: https://doi.org/10.5114/jos.2021.106571

\section{INTRODUCTION}

Periodontitis (PD) is a common disease, which might lead to tooth loss and poses many challenges for clinicians. During periodontitis, inflammation of tooth supporting tissues results in progressive destruction of periodontal ligaments and alveolar bones [1]. The goal of PD treatment is to eliminate bacteria, reduce inflammation, improve aesthetics, and maintain the function of periodontal tissue. Among the phases of treatment, the non-surgical phase (scaling and root planning - SRP) is usually the first and indispensable one. However, this phase is not effective
JOURNAL OF STOMATOLOGY CZASOPISMO STOMATOLOGICZNE
AdDresS For CORRESPONDENCE: Nam Cong-Nhat Huynh, Department of Dental Basic Sciences, Faculty of Odonto-Stomatology, University of Medicine and Pharmacy at Ho Chi Minh City, Vietnam, 652 Nguyen Trai, W11, D5, 749000, Ho Chi Minh city, Viet Nam, e-mail: namhuynh@ump.edu.vn

ReCeIved: 26.01.2021 • ACCEPTED: 27.03.2021 • Published: 04.06.2021 
in cases of periodontal pockets with more than $5 \mathrm{~mm}$ in depth, or with furcation involvement $[1,2]$.

Recently, to promote healing process and enhance the effect of conventional SRP, antimicrobial and healing support agents have been applied topically in the periodontal pockets, such as tetracycline, metronidazole, minocycline, chlorhexidine, doxycycline, and plasma rich in growth factors $[3,4]$. Many studies have documented the clinical efficacy of SRP in combination with local agent delivery, which improved the effectiveness of treatment, reduced the frequency of surgical phase, and treatment cost for patients $[2,3,5]$. One of the materials is hyaluronic acid (HA) [6]. HA has long been studied and applied in many fields of medicine [7]. HA is a polysaccharide and plays many important roles in maintaining stability, adjusting the viscosity of biological fluids, homeostasis, adjusting osmotic pressure, and supporting structures. HA can be derived from bacteria or animals. This is a versatile, non-toxic, and nonimmunogenic biological material as well as highly biocompatible [8]. The wide application of HA in medicine is due to its capability of binding multiple water molecules, improving tissue hydration, and preventing mechanical damage; it presents bacteriostatic, antiinflammatory, and osteo-inductive proprieties $[6,7]$.

\section{OBJECTIVES}

The aim of this study was to assess the clinical effects of subgingival application of $0.2 \% \mathrm{HA}$ gel, following scaling and root planning (SRP) in PD patients. This study was the first to be conducted in Vietnam to evaluate the effect of HA on the response of periodontal tissue and its bactericidal efficiency in chronic periodontitis. Porphyromonas gingivalis (Pg), Treponema denticola (Td), Fusobacterium nucleatum (Fn), and Tannerella forsythia ( $T f$ ) bacteria were studied, which were important species in the development of periodontitis $[1,9,10]$. The research results can supplement scientific evidence about the effects of this compound, and clarify the application of HA in clinical periodontitis treatment.

\section{MATERIAL AND METHODS}

\section{TRIAL DESIGN}

A randomized, controlled clinical trial was performed using a split-mouth design. Study procedure consisted of information collecting, clinical measurements, and data analysis. The study was conducted in full accordance with the World Medical Association Declaration of Helsinki 2000, and approved by the Institutional Review Board and Ethics Committee at the University of Medicine and Pharmacy in Ho Chi Minh City, Vietnam (approval number, 312/DHYD-HDDD). A signed informed consent was obtained from every participant before enrollment.

\section{ELIGIBILITY CRITERIA}

Participants who came for periodontitis treatment were recruited at the Department of Periodontology, Faculty of Odonto-Stomatology. Inclusion criteria concerned patients 18 years and older, with a minimum number of 20 teeth, with at least 3 teeth in the contralateral quadrants diagnosed with moderate or severe periodontitis according to the 2015 American Academy of Periodontology (AAP) guidelines: gingival bleeding at examination, $\geq 5 \mathrm{~mm}$ periodontal pocket depth, bone resorption on X-ray film $\geq 16 \%$, or $>3 \mathrm{~mm}$ root length [11-13]. Patients who were allergic to ingredients, being pregnant/ lactating, smoking, alcoholic, who had undergone periodontal therapy in the last 6 months, received antibiotic therapy, anti-inflammatory agents, statins, drug-induced gingival enlargement (phenytoin, cyclosporin, or nifedipine) in the last 3 months, were excluded. Moreover, patients with systemic diseases, such as cardiovascular disease, hypertension, diabetes mellitus, or orthodontic treatment, and patients who refused to participate in the study and/or follow-up visits were also excluded.

\section{SAMPLE SIZE}

The sample size was calculated using the following formula:

$$
N=\left[\frac{Z_{1-\frac{\alpha}{2}}+Z_{1-\beta}}{\mu_{1}-\mu_{2}}\right]^{2} \times\left(\sigma_{1}^{2}+\sigma_{2}^{2}\right)
$$

According to Rajan [11], to obtain the difference of probing pocket depth (PPD) of the two groups (control and $\mathrm{HA}$ ), $\mu_{1}=4.09$ and $\mu_{2}=3.21$ were the average PPDs, and $\sigma_{1}=1.38$ and $\sigma_{2}=0.65$ were standard deviation of the two groups, respectively. With $\alpha=0.05$ and $\beta=0.2$, we calculated $Z_{\frac{\alpha}{2}}=1.96$ and $Z \beta=0.84$, then $N=25$. To compensate for sample loss, 34 subjects were enrolled in this study.

\section{RANDOMIZATION AND BLINDING}

Randomized assignment of patients' each group was determined using sealed envelopes by an investigator who was blinded to all patients' information, following analysis, and evaluation of the data. The details of the sequence were concealed from all clinicians who participated in the study. Blinding was ensured because an independent investigator administered HA during subsequent visits, while the patients, periodontist, and outcome assessing investigators were unaware of the intervention given to the test sites.

\section{INTERVENTIONS}

\section{SUBGINGIVAL CALCULUS SCALING AND ROOT PLANING}

After periodontal examination and medical history evaluation, all patients received oral hygiene instruction 
and advanced periodontal treatment intervention at T0 (time point 0 ), consisting of full mouth subgingival SRP with ultrasonic scalers (Cavitron Jet Plus, Dentsply Sirona, Mississauga, Canada) and Gracey curettes ( $\mathrm{Hu}-$ Friedy Manufacturing, Chicago, USA).

\section{APPLICATION OF HYALURONIC ACID IN PERIODONTAL POCKETS}

After supragingival SRP, periodontal pockets were rinsed by $0.9 \% \mathrm{NaCl}$ solution. The experimental sites were then gently dried by air blow and isolated with cotton rolls. Then, $1 \mathrm{ml}$ of $0.2 \%$ HA gel (GENGIGEL ${ }^{\oplus}$, Ricerfarma srl, Milano, Italy) was injected to the bottom of each pocket. The gingiva was not covered after HA application and the patients were required not to drink or rinse within 30 minutes. The procedure was applied at $\mathrm{T} 0$ and the $1^{\text {st }}, 2^{\text {nd }}$, and $3^{\text {rd }}$ week recalls [14]. Full-mouth polishing was performed at the $3^{\text {rd }}$ week recall after HA application.

\section{OUTCOMES}

\section{PERIODONTAL CLINICAL PARAMETERS}

Periodontal disease assessment was conducted before SRP (T0) and after 6 weeks (T6), as in our previous studies [15]. Plaque index (PLI), gingival index (GI), probing pocket depth (PPD), clinical attachment loss (CAL), and bleeding on probing (BOP) were evaluated at six positions in all teeth (mesio-buccal, buccal, disto-buccal, mesio-lingual, lingual, and disto-lingual). The average values of PLI, GI, PPD, and CAL were calculated for each tooth. BOP was presented by the percentage of recorded bleeding sites. The inter-rater reliability assessment was conducted as follows: the periodontal parameters of 5 participants, with a total of 133 teeth were evaluated by the rater on a continuous scale; after one hour, the measurement was repeated under the same condition. The inter-class correlation coefficient (ICC) was determined as $85.9 \%, 84.02 \%, 83.08 \%$, $80.64 \%$, and $85.15 \%$ for PLI, GI, PPD, CAL, and BOP, respectively.

\section{SUBGINGIVAL PLAQUE COLLECTING AND REAL-TIME POLYMERASE CHAIN REACTION (PCR)}

Subgingival plaques collecting for bacteria evaluation was performed before SRP (T0) and after 6 weeks. Subgingival plaques were obtained by a sterile paper point (No. 30) at the deepest pocket in contralateral quadrants. Firstly, the tooth was removed from supragingival plaque, isolated with cotton rolls, and air-dried. Then, the paper point was inserted into the bottom of the periodontal pocket [16]. After 30 seconds, the point was removed and placed into a $1.5 \mathrm{ml}$ Eppendorf tube containing phosphate buffered saline (PBS). The tube was kept in an iced box and transferred to the laboratory within 4 hours. Real-time PCR was performed to detect the number of $P g, T d, F n$, and Tf. Briefly, bacterial deoxyribonucleic acid (DNA) extraction was prepared by centrifuging the samples at 9300 revolutions per minute (rpm), for 3 minutes. After removing PBS, precipitants were added with $200 \mu$ of tris-ethylene diamine tetraacetic acid (Tris-EDTA) buffer to extract DNA using a real-time PCR machine (CFX96 Real-Time PCR System, Bio-Rad, Hercules, CA, USA). Then, bacterial load was calculated as $\log _{10}$ copies/ml and compared between the groups.

\section{STATISTICAL ANALYSIS}

Statistical analyses were performed in Stata, version 13.0 for Windows (Stata Corp., College Station, Texas, USA). Shapiro-Wilk test was used to examine normality distribution of the outcome variables. We applied $\chi^{2}$ test for categorical variables, and Mann-Whitney $U$-test for continuous variables. Wilcoxon signed-rank test was used to compare repeated measurements in each group, and the Mann-Whitney $U$-test was applied to assess statistical differences in means between the two groups. A $p$-value of less than 0.05 was set as statistically significant.

\section{RESULTS}

The flow diagram of the study is shown in Figure 1. In total, 34 patients were included in the study. Six patients were excluded, with 2 patients using antibiotics during the study and 4 patients who did not control plaque as per the requirements; therefore, the results of 28 patients ( 6 males, 22 females) were used for data analysis. 733 periodontal pockets were sampled, including 313 slightly deep pockets ( $>3 \mathrm{~mm}$ and $<5 \mathrm{~mm}$ ), 308 moderately deep pockets ( $\geq 5 \mathrm{~mm}$ and $<7 \mathrm{~mm}$ ), and 112 deep pockets $(\geq 7 \mathrm{~mm}$ ) (Figure $2 \mathrm{~A}$ ).

\section{PERIODONTAL CLINICAL PARAMETERS (TABLE 1)}

Within each group, there was a significant decrease in the mean PLI, GI, BOP, PPD, and CAL from baseline to $6^{\text {th }}$ week $(p<0.001)$. Between the two groups, no statistically significant difference was observed in the mean GI and BOP at baseline $(p>0.05)$. However, there was a statistically significant reduction in the GI and BOP in the test group, as compared to the control group at $6^{\text {th }}$ week $(p<0.05)$. There was no statistically significant difference in the mean PLI, PPD, and CAL in the two groups at both baseline and $6^{\text {th }}$ week $(p>0.05)$. 


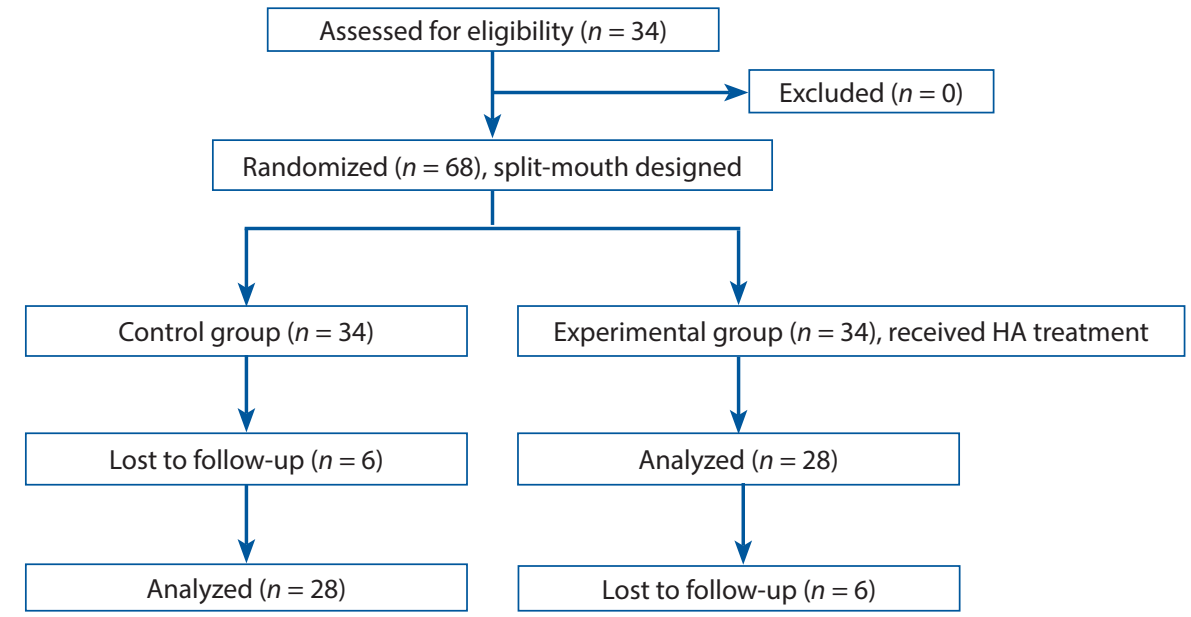

FIGURE 1. Flow diagram of the study

\section{PROBING POCKET DEPTH AND CLINICAL ATTACHMENT LOSS IN MODERATELY DEEP POCKETS \\ $(5 \mathrm{MM} \leq \mathrm{PPD}<7 \mathrm{MM})$}

Figure $2 \mathrm{~B}$ shows that at baseline, no statistically significant difference was observed in the mean PPD and CAL in the test and control groups at baseline $(p>0.05)$. At the $6^{\text {th }}$ week, there was a significant reduction in the PPD and CAL in the test group, as compared to the control group $(p<0.05)$.

\section{PROBING POCKET DEPTH AND CLINICAL ATTACHMENT LOSS IN DEEP POCKETS (PPD $\geq 7 \mathrm{MM})$}

Figure 2C indicates that there was a decrease in the mean PPD and CAL from baseline to $6^{\text {th }}$ week, but there was no statistical difference found $(p>0.05)$.

\section{BACTERIA IN SUBGINGIVAL PLAQUE}

Between the two groups, no statistically significant difference was noted in the mean bacterial number of $\mathrm{Td}$ and $\mathrm{Pg}$ in the two groups at both baseline and $6^{\text {th }}$ week $(p>0.05)$ (Table 2$)$. In the test group, the mean bacterial number $T f$ at $6^{\text {th }}$ week was significantly lower than in the control group, and no statistical difference was indicated in the two groups at baseline.

In the control group, no statistically significant difference was observed in the mean number of $F n$ at baseline and $6^{\text {th }}$ week $(p>0.05)$. However, in the test group, there was a statistically significant reduction in the mean number of $F n$ at $6^{\text {th }}$ week, as compared to baseline $(p<0.05)$.

In both the groups, there was statistically significant reduction in the mean number of 3 types of bacteria ( $T d, T f$, and $P g$ ) at the $6^{\text {th }}$ week, as compared to baseline $(p<0.05)$. In the control group, no statistically signifi-
TABLE 1. Periodontal clinical parameters between two groups before and after treatment with hyaluronic acid

\begin{tabular}{|c|c|c|c|}
\hline Parameters/Time & Control group & Test group & $\boldsymbol{p}^{*}$ \\
\hline \multicolumn{4}{|l|}{ Plaque index } \\
\hline Baseline & $0.97 \pm 0.71$ & $0.91 \pm 0.71$ & 0.070 \\
\hline 6 weeks & $0.41 \pm 0.53$ & $0.30 \pm 0.51$ & 0.080 \\
\hline$p^{\dagger}$ & $<0.001$ & $<0.001$ & \\
\hline \multicolumn{4}{|l|}{ Gingival index } \\
\hline Baseline & $1.81 \pm 0.42$ & $1.78 \pm 0.44$ & 0.230 \\
\hline 6 weeks & $0.87 \pm 0.72$ & $0.23 \pm 0.45$ & $<0.001$ \\
\hline$p^{\dagger}$ & $<0.001$ & $<0.001$ & \\
\hline \multicolumn{4}{|l|}{ Bleeding on probing } \\
\hline Baseline & 68.15 & 62.17 & 0.120 \\
\hline 6 weeks & 20.37 & 13.39 & 0.009 \\
\hline$p^{\dagger}$ & $<0.001$ & $<0.001$ & \\
\hline \multicolumn{4}{|c|}{ Periodontal pocket depth } \\
\hline Baseline & $3.18 \pm 1.59$ & $3.31 \pm 1.81$ & 0.310 \\
\hline 6 weeks & $2.41 \pm 1.32$ & $2.33 \pm 1.27$ & 0.070 \\
\hline$p^{\dagger}$ & $<0.001$ & $<0.001$ & \\
\hline \multicolumn{4}{|c|}{ Clinical attachment loss } \\
\hline Baseline & $3.68 \pm 1.69$ & $3.78 \pm 1.96$ & 0.060 \\
\hline 6 weeks & $2.97 \pm 1.69$ & $2.88 \pm 1.49$ & 0.140 \\
\hline$p^{\dagger}$ & $<0.001$ & $<0.001$ & \\
\hline
\end{tabular}

cant difference in the mean number of $F n$ was noted at $6^{\text {th }}$ week, as compared to baseline $(p>0.05)$.

\section{DISCUSSION}

Evaluation of clinical parameters (BOP, CAL, PDD, GI, and PLI) plays an important role in periodontitis 
A

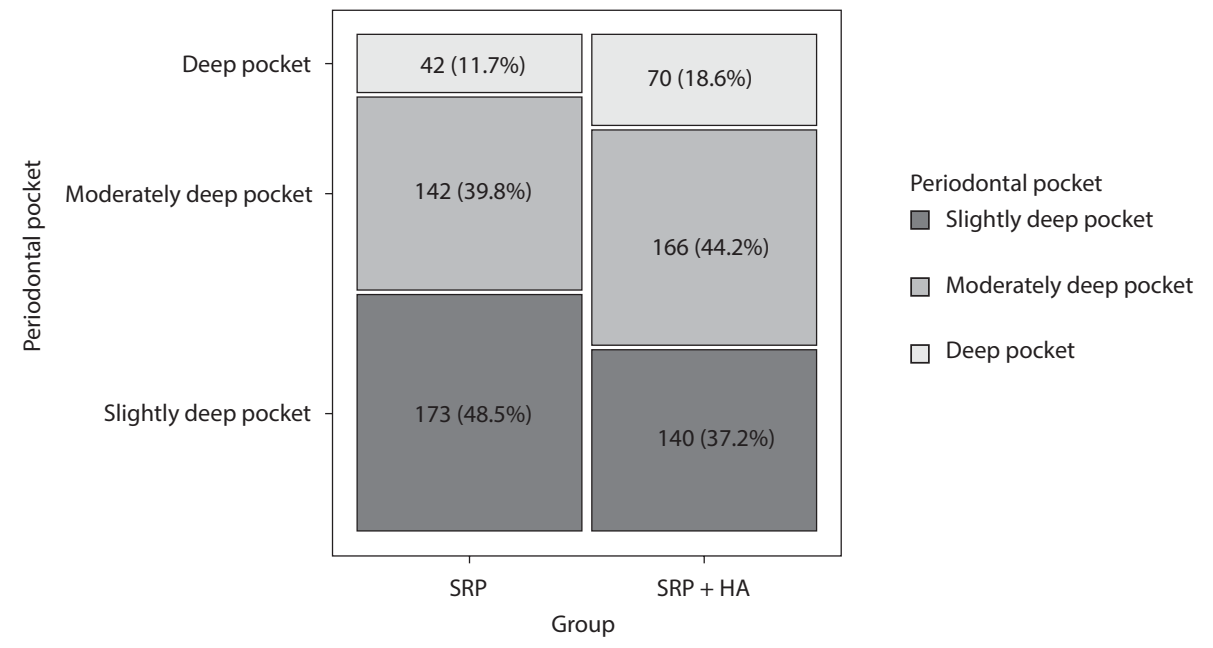

B
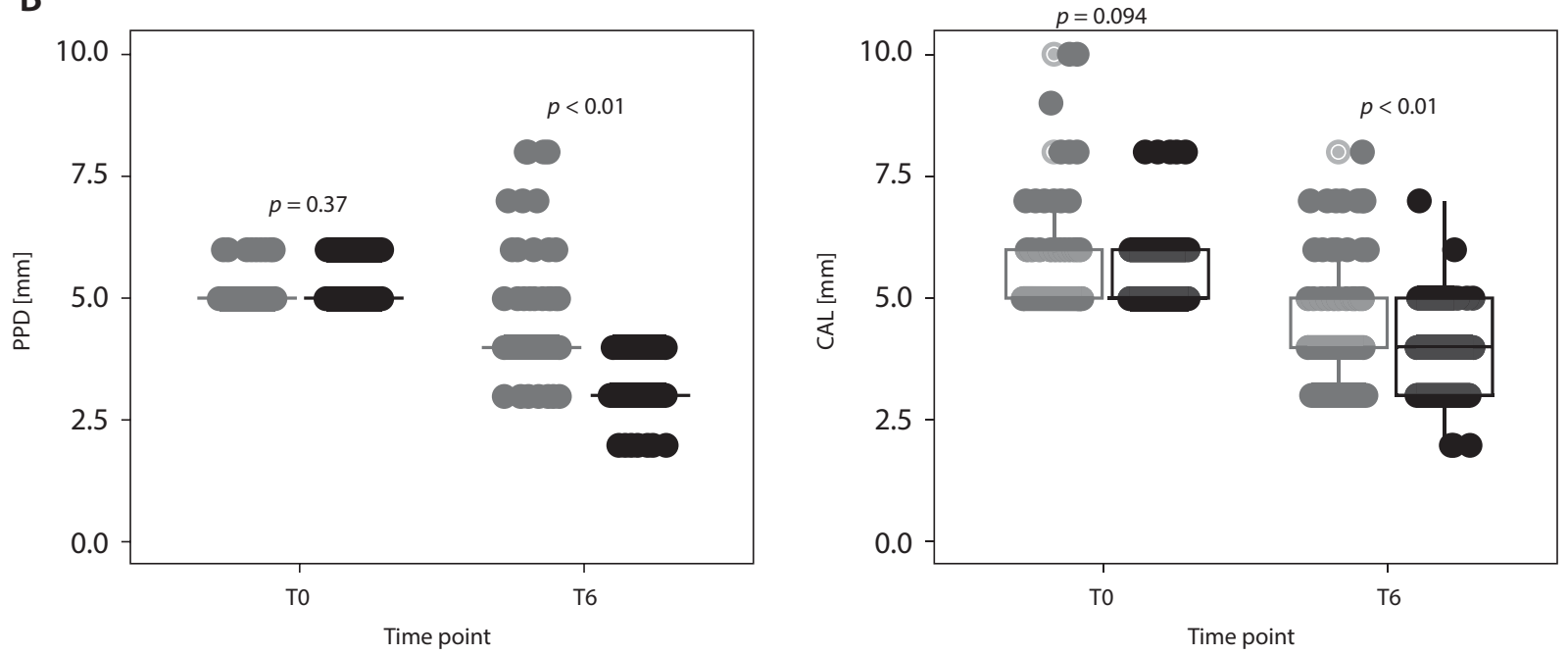

C
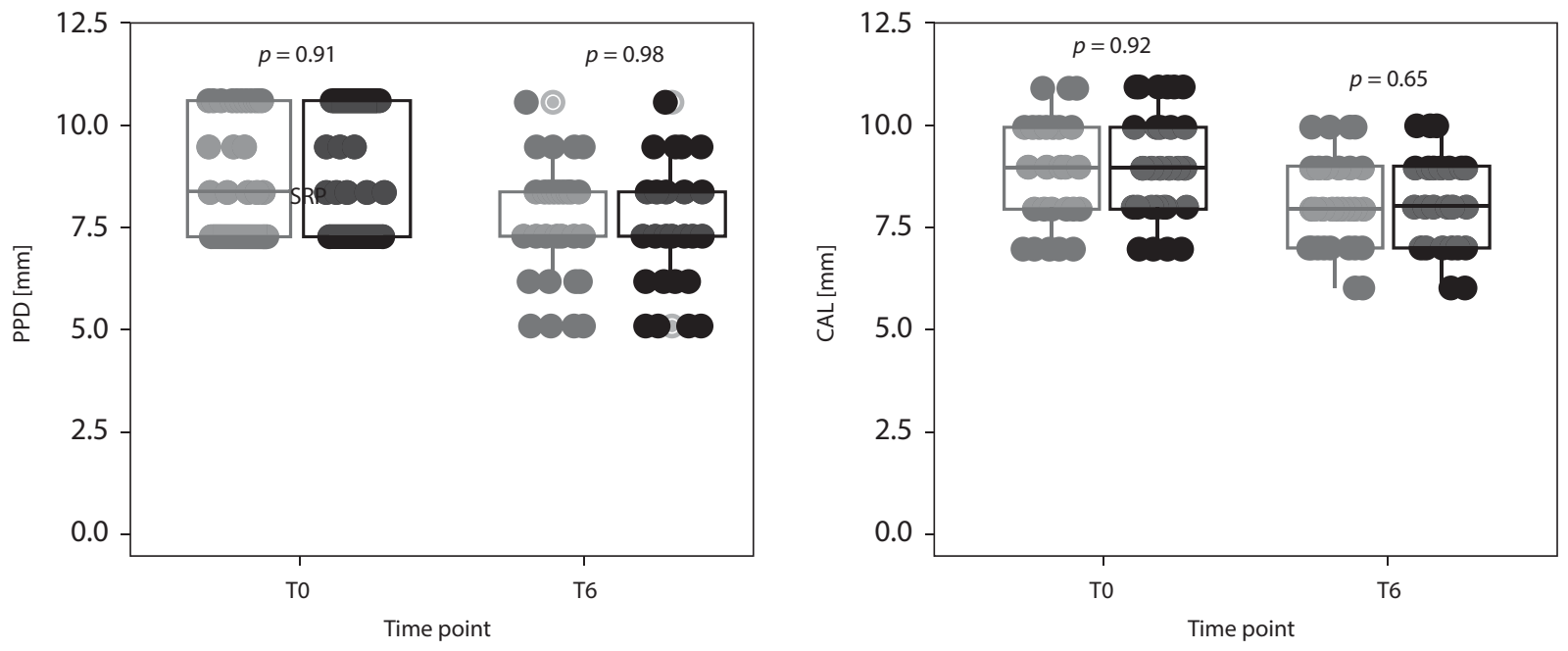

Group

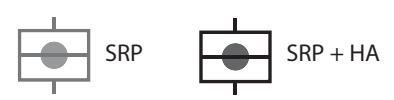

FIGURE 2. A) Periodontal pocket number in both the groups at baseline. B) Periodontal pocket depth (PPD) and clinical attachment loss (CAL) measurement in the moderately deep pockets. C) PPD and CAL measurement in the deep pockets (Mann-Whitney test) 
diagnosis. However, these indicators only show retrospective information, i.e., consequences of the disease and extent of the destruction, rather than current status or risk of disease progression in the future. PD is a disease caused by bacteria; therefore, the analysis of bacteria would help in detection and evaluation of the condition of the disease, predicting disease progression, and evaluating the effectiveness of patient's response to treatment methods. Hence, in addition to evaluating clinical indicators, we performed subgingival plaque collection to assess bacteria before and after the treatment between the two groups. The split-mouth study design helped to reduce the effects of confounding factors on age, gender, and level of plaque control of the study participants.

There are many techniques used to detect and quantify bacteria, including bacterial culture, chromogenic media, microscopy techniques, and biochemical and molecular methods [17]. Culture media and biochemical tests are known to be very time-consuming and labor-intensive [17]. The real-time-PCR technique is a highly specific molecular biology technique that delivers reliable results $[18,19]$.

A statistically significant reduction in all periodontal clinical parameters after the local subgingival application of the HA in both the groups was observed in our study. SRP is indispensable in most of PD cases, to remove calculus and plaque, bacteria toxins, and to establish an acceptable root surface [20,21]. SRP with or without supplementation of HA helped to improve periodontal condition. There was no statistically significant difference in the mean GI and BOP between the two groups at baseline; after 6 weeks, the mean GIs and BOPs in the test group were significantly lower than that in the control cohort. This result was similar to the report of Gontiya [14], Polepalle [5], and Omer [22]. Subgingival placement of HA gel after SRP was shown to provide a significant improvement in gingivitis parameters. $0.2 \%$ HA gel has been demonstrated to have a beneficial effect in the treatment of plaque-induced gingivitis and in inflammation and gingival bleeding control [7], which may be due to the action as a scavenger by draining prostaglandins, metalloproteinases, and other bio-active molecules [23].

Between the groups, no statistically significant difference was shown in the mean PLI, PPD, and CAL at both baseline and $6^{\text {th }}$ week $(p>0.05)$. This result was similar to the report of Gontiya [14] and Polepalle [5]. There was a significant reduction in PPD and CAL of moderately deep pockets in the test group as compared to the control group, which could be explained by the fact that HA has comparable roles in the healing of mineralized and non-mineralized tissues of the periodontium $[24,25]$. Therefore, HA is suggested to be helpful in improving periodontal pocket and clinical attachment in the moderately deep pockets.

In both the groups, there was a decrease in the mean PPD and CAL of deep pockets from baseline to $6^{\text {th }}$ week,
TABLE 2. Bacterial load of Treponema denticola, Fusobacterium nucleatum, Tannerella forsythia, and Porphyromonas gingivalis in two groups before and after treatment

\begin{tabular}{|c|c|c|c|}
\hline Mean*/Time & Control group & Test group & $p^{* *}$ \\
\hline \multicolumn{4}{|c|}{ Treponema denticola } \\
\hline Baseline & $4.43 \pm 3.06$ & $4.15 \pm 3.01$ & 0.510 \\
\hline 6 weeks & $3.22 \pm 2.97$ & $2.10 \pm 2.72$ & 0.190 \\
\hline$p^{\dagger}$ & 0.030 & 0.017 & \\
\hline \multicolumn{4}{|c|}{ Fusobacterium nucleatum } \\
\hline Baseline & $5.67 \pm 1.23$ & $5.32 \pm 2.23$ & 0.490 \\
\hline 6 weeks & $5.60 \pm 1.33$ & $4.16 \pm 2.45$ & 0.110 \\
\hline$p^{\dagger}$ & 0.080 & 0.019 & \\
\hline \multicolumn{4}{|c|}{ Tannerella forsythia } \\
\hline Baseline & $5.96 \pm 1.90$ & $5.57 \pm 1.96$ & 0.280 \\
\hline 6 weeks & $5.08 \pm 1.64$ & $3.43 \pm 2.76$ & 0.030 \\
\hline$p^{\dagger}$ & 0.020 & 0.005 & \\
\hline \multicolumn{4}{|c|}{ Porphyromonas gingivalis } \\
\hline Baseline & $3.96 \pm 3.18$ & $4.09 \pm 2.82$ & 0.680 \\
\hline 6 weeks & $2.77 \pm 2.53$ & $2.00 \pm 2.66$ & 0.200 \\
\hline$p^{\dagger}$ & $<0.001$ & 0.010 & \\
\hline
\end{tabular}

${ }^{*} \log _{10}$ copies $/ \mathrm{ml} ;{ }^{* *}$ Mann-Whitney test; ${ }^{+}$Wilcoxon sign-rank test

but no statistical difference was found. This result may be due to complicated root anatomy or furcation involvement, difficult root planning, and consequently, incomplete subgingival calculus removing [26]. The results suggest that surgical therapy is indicated in the treatment of deep pockets. In addition, the number of deep periodontal pockets in the present study was not large enough; therefore, the outcomes should be interpreted with caution. It is necessary to investigate a larger number of periodontal pockets in order to assess the precise difference between both the groups.

In our study, we collected and quantified the bacteria of gingival plaque located in the deepest periodontal pocket of each group by using real-time PCR. This is considered a reliable, fast, and accurate method of quantifying bacteria $[19,27]$. The numbers of bacteria in the two groups were not statistically different at both times. In each group, there was a decrease in the number of all 3 types of bacteria ( $T d, T f$, and $P g$ ) after the intervention compared to baseline, with the difference being statistically significant. This result was similar to the study report of Sigrun [12] and Xu [28]. The number of $F n$ in the control group decreased after the treatment, but the difference was not statistically significant, while the decrease of $F n$ in the test group was statistically significant. In the test group, the mean bacterial number $T f$ at $6^{\text {th }}$ week was significantly lower than SRP group, while no significant difference was shown in both the groups 
at baseline. We believe that the above change is due to specificity of periodontitis location and the subgingival plaque collected at the deepest place, which was not representative enough. In addition, it may be because HA is highly effective in the medium periodontal pockets rather than the deep ones, where the plaques for bacteria analysis were collected.

HA is considered safe to living cells; however, some side effects, though not serious, have been reported. HA may cause bruising, swelling, redness, pain, itching, and pain at the sites, mainly where the injection was applied [29]. Therefore, in order to reduce such unfavorable reactions in our patients, we applied $0.2 \% \mathrm{HA}$ instead of $0.8 \% \mathrm{HA}$, as some authors have used it in theirs' studies. In the present research, the antibacterial mechanism of HA has not been clearly demonstrated. A possible explanation for this result could be that the efficacy of improving gingivitis and clinical parameters in the moderate periodontal pockets is regulated by some immunomodulatory or unknown mechanisms. Further studies are needed to clarify the mechanism of HA in periodontal tissues.

\section{CONCLUSIONS}

Within the limitation of this study, the application of HA gel to periodontal pockets during non-surgical periodontitis treatment significantly improved gingivitis, reduced pocket depth, and increased clinical attachment level in the medium periodontal pockets. Moreover, this additional treatment appeared to help reducing 2 types of bacteria ( $T f$ and $F n$ ) better than non-surgical treatment only.

\section{ACKNOWLEDGEMENTS}

This work was granted in part by the University of Medicine and Pharmacy at Ho Chi Minh City, with No. 64/2019/HD-DHYD.

\section{CONFLICT OF INTEREST}

The authors declare no potential conflicts of interest with respect to the research, authorship, and/or publication of this article.

\section{References}

1. Newman M, Takei H, Klokkevold PR, Carranza FA. Newman and Carranza's Clinical Periodontology. $13^{\text {th }}$ ed. Amsterdam: Elsevier Health Sciences; 2018.

2. Research, Science and Therapy Committee of the American Academy of Periodontology. Treatment of plaque-induced gingivitis, chronic periodontitis, and other clinical conditions. J Periodontol 2001; 72: 1790-1800.

3. Greenstein G, Tonetti M. The role of controlled drug delivery for periodontitis. The Research, Science and Therapy Committee of the American Academy of Periodontology. J Periodontol 2000; 71: 125-140.

4. Panda S, Purkayastha A, Mohanty R, et al. Plasma rich in growth factors (PRGF) in non-surgical periodontal therapy: a randomized clinical trial. Braz Oral Res 2020; 34: e034.

5. Polepalle T, Srinivas M, Swamy N, et al. Local delivery of hyaluronan $0.8 \%$ as an adjunct to scaling and root planing in the treatment of chronic periodontitis: a clinical and microbiological study. J Indian Soc Periodontol 2015; 19: 37-42.

6. Eliezer M, Imber JC, Sculean A, et al. Hyaluronic acid as adjunctive to non-surgical and surgical periodontal therapy: a systematic review and meta-analysis. Clin Oral Invest 2019; 23: 3423-3435.

7. Dahiya P, Kamal R. Hyaluronic acid: a boon in periodontal therapy. N Am J Med Sci 2013; 5: 309-315.

8. Mueller A, Fujioka-Kobayashi M, Mueller HD, et al. Effect of hyaluronic acid on morphological changes to dentin surfaces and subsequent effect on periodontal ligament cell survival, attachment, and spreading. Clin Oral Invest 2017; 21: 1013-1019.

9. Sela MN. Role of Treponema denticola in periodontal diseases. Crit Rev Oral Biol Med 2001; 12: 399-413.

10. Rafiei M, Kiani F, Sayehmiri F, Sayehmiri K, Sheikhi A, Zamanian Azodi M. Study of Porphyromonas gingivalis in periodontal diseases: A systematic review and meta-analysis. Med J Islam Repub Iran 2017; 31: 62.

11. Rajan P, Baramappa R, Rao NM, et al. Hyaluronic acid as an adjunct to scaling and root planing in chronic periodontitis. A randomized clinical trail. J Clin Diagn Res 2014; 8: ZC11-14.

12. Eick S, Renatus A, Heinicke M, et al. Hyaluronic acid as an adjunct after scaling and root planing: a prospective randomized clinical trial. J Periodontol 2013; 84: 941-949.

13. American Academy of Periodontology. American Academy of Periodontology Task Force Report on the Update to the 1999 Classification of Periodontal Diseases and Conditions. J Periodontol 2015; 86: 835-838.

14. Gontiya G, Galgali SR. Effect of hyaluronan on periodontitis: a clinical and histological study. J Indian Soc Periodontol 2012; 16: 184-192.

15. Nguyen VB, Nguyen TT, Huynh NC, et al. Relationship between periodontitis and rheumatoid arthritis in Vietnamese patients. Acta Odontol Scand 2020; 78: 522-528.

16. Al-Shammari NM, Shafshak SM, Ali MS. Effect of $0.8 \%$ hyaluronic acid in conventional treatment of moderate to severe chronic periodontitis. J Contemp Dent Pract 2018; 19: 527-534.

17. Franco-Duarte R, Černáková L, Kadam S, Kaushik KS, Salehi B, Bevilacqua A, et al. Advances in chemical and biological methods to identify microorganisms - from past to present. Microorganisms 2019; 7: 130

18. Lyons SR, Griffen AL, Leys EJ. Quantitative real-time PCR for Porphyromonas gingivalis and total bacteria. J Clin Microbiol 2000; 38: 2362-2365.

19. Nozaki T, Kusumoto Y, Kitamura M, Hirano H, Kohyama A, Hayakawa M, et al. A sensitive method for detecting Porphyromonas gingivalis by polymerase chain reaction and its possible clinical application. J Periodontol 2001; 72: 1228-1235.

20. Kumar P, Das SJ, Sonowal ST, Chawla J. Comparison of root surface roughness produced by hand instruments and ultrasonic scalers: an invitro study. J Clin Diagn Res 2015; 9: Zc56-60.

21. Yaghini J, Naghsh N, Attaei E, Birang R, Birang E. Root surface roughness after scaling and root planing with Er:YAG laser compared to hand and ultrasonic instruments by profilometry. J Dent (Tehran) 2015; 12: 899-905.

22. Basheer O, Asim S, Bakri G, Nada H. The effect of local application of hyaluronan gel as an adjunctive to scaling and root planing in patients. African Journal of Dentistry 2018; 6: 163-170.

23. Laurent TC, Laurent UB, Fraser JR. Functions of hyaluronan. Ann Rheum Dis 1995; 54: 429-432.

24. Bertl K, Bruckmann C, Isberg PE, Klinge B, Gotfredsen K, Stavropoulos A. Hyaluronan in non-surgical and surgical periodontal therapy: a systematic review. J Clin Periodontol 2015; 42: 236-246. 
25. Casale M, Moffa A, Vella P, et al. Hyaluronic acid: perspectives in dentistry. A systematic review. Int J Immunopathol Pharmacol 2016; 29: 572-582

26. Van der Weijden GAF, Dekkers GJ, Slot DE. Success of nonsurgical periodontal therapy in adult periodontitis patients a retrospective analysis. Int J Dent Hyg 2019; 17: 309-317.

27. Göhler A, Hetzer A, Holtfreter B, et al. Quantitative molecular detection of putative periodontal pathogens in clinically healthy and periodontally diseased subjects. PLoS One 2014; 9: e99244.

28. Xu Y, Höfling K, Fimmers R, Frentzen M, Jervøe-Storm PM. Clinical and microbiological effects of topical subgingival application of hyaluronic acid gel adjunctive to scaling and root planing in the treatment of chronic periodontitis. J Periodontol 2004; 75 1114-1118.

29. Bansal J, Kedige SD, Anand S. Hyaluronic acid: a promising mediator for periodontal regeneration. Indian J Dent Res 2010; 21: 575-578. 